\title{
Overexpression of CENPF correlates with poor prognosis and tumor bone metastasis in breast cancer
}

Jingbo Sun ${ }^{1 \dagger}$, Jingzhan Huang ${ }^{1 \dagger}$, Jin Lan ${ }^{1 \dagger}$, Kun Zhou $^{1}$, Yuan Gao ${ }^{1}, Z_{\text {Zhigao Song }}^{2}$, Yunyao Deng ${ }^{1}$, Lixin Liu ${ }^{{ }^{*}}$, Ying Dong ${ }^{3^{*}}$ and Xiaolong Liu ${ }^{1^{*}}$ (D)

\begin{abstract}
Background: Centromere Protein F (CENPF) associates with the centromere-kinetochore complex and influences cell proliferation and metastasis in several cancers. The role of CENPF in breast cancer (BC) bone metastasis remains unclear.

Methods: Using the ONCOMINE database, we compared the expression of CENPF in breast cancer and normal tissues. Findings were confirmed in $60 \mathrm{BC}$ patients through immunohistochemical (IHC) staining. Microarray data from GEO and Kaplan-Meier plots were used analyze the overall survival (OS) and relapse free survival (RFS). Using the GEO databases, we compared the expression of CENPF in primary lesions, lung metastasis lesions and bone metastasis lesions, and validated our findings in BALB/C mouse 4T1 BC models. Based on gene set enrichment analysis (GSEA) and western blot, we predicted the mechanisms by which CENPF regulates $B C$ bone metastasis.

Results: The ONCOMINE database and immunohistochemical (IHC) showed higher CENPF expression in BC tissue compared to normal tissue. Kaplan-Meier plots also revealed that high CENPF mRNA expression correlated to poor survival and shorter progression-free survival (RFS). From BALB/C mice 4T1 BC models and the GEO database, CENPF was overexpressed in primary lesions, other target organs, and in bone metastasis. Based on gene set enrichment analysis (GSEA) and western blot, we predicted that CENPF regulates the secretion of parathyroid hormone-related peptide (PTHrP) through its ability to activate PI3K-AKT-mTORC1.
\end{abstract}

Conclusion: CENPF promotes BC bone metastasis by activating PI3K-AKT-mTORC1 signaling and represents a novel therapeutic target for $\mathrm{BC}$ treatment.

Keywords: CENPF, Breast cancer, Bone metastasis, mTORC1

\section{Background}

Breast cancer $(\mathrm{BC})$ remains a leading cause of cancer related death in women across the globe [1]. In total, $60 \%$ to $75 \%$ of metastasis in BC leads to bone metastasis

\footnotetext{
*Correspondence: I|x0129@i.smu.edu.cn; 240201053@qq.com; Ixl1979@i. smu.edu.cn

${ }^{\dagger}$ Jingbo Sun, Jingzhan Huang and Jin Lan contributed equally to the study

${ }^{1}$ Department of General Surgery, The Third Affiliated Hospital of Southern Medical University, 183 West Zhongshan Avenue, Guangzhou 510630, Guangdong, China

${ }^{3}$ Nursing Department, The Third Affiliated Hospital of Southern Medical University, 183 West Zhongshan Avenue, Guangzhou 510630, Guangdong, China

Full list of author information is available at the end of the article
}

(BM) [2]. Bone metastasis impair the quality of life due to hypercalcemia, bone pain, fractures, nerve compression, a reduction in mobility, and reduced social function $[3,4]$. When bone metastasis occurs, the disease enters an incurable stage, with a median survival time of only 2 years, and 5-year survival rates of 20\% [5-8]. Controlling bone metastasis in breast cancer remains a problem in clinical practice.

Bone metastasis is a complex, multistage process that includes local invasion, intravasation, survival in the circulation, extravasation, and colonization $[9,10]$. An array of pathogenic molecules mediate $\mathrm{BC}$ bone metastasis including parathyroid hormone-related protein (PTHrP), interleukin 8 (IL-8), and vascular cell adhesion molecule

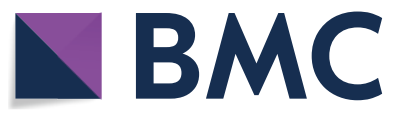

(c) The Author(s) 2019. This article is distributed under the terms of the Creative Commons Attribution 4.0 International License (http://creativecommons.org/licenses/by/4.0/), which permits unrestricted use, distribution, and reproduction in any medium, provided you give appropriate credit to the original author(s) and the source, provide a link to the Creative Commons license, and indicate if changes were made. The Creative Commons Public Domain Dedication waiver (http://creativecommons.org/ publicdomain/zero/1.0/) applies to the data made available in this article, unless otherwise stated. 
1 (VCAM-1) [11-13]. Despite progress in the molecular basis of bone metastasis in $\mathrm{BC}$, knowledge of the mechanisms underlying this process are required to identify targets for the prevention and treatment of $\mathrm{BC}$.

The Centromere Protein F (CENPF) is a cell cycleassociated nuclear antigen that is expressed to low levels in G0/G1-cells and accumulates in the nuclear matrix during the S-phase, with maximal expression in G2/M-cells. CENPF was identified as a marker of cell proliferation in several human malignancies, including BC [14] and is overexpressed in hepatocellular carcinoma (HCC) [15] and other tumors [16]. Additionally, elevated CENPF expression contributes to unregulated cell proliferation in HCC [17]. It was recently shown that CENPF and FOXM1 are synergistic master regulators of prostate cancer malignancy and are prognostic indicators of poor survival and metastasis [18]. Furthermore, COUP transcription factor 2 (COUP-TFII) promotes metastasis in prostatic cancer $(\mathrm{PC})$ through CENPF signaling [19].

In this study, we demonstrate that CENPF is a valuable prognostic predictor of $\mathrm{BC}$. Bioinformatics and computational analysis demonstrated that CENPF regulates $\mathrm{BC}$ metastasis to bone through PI3K-AKT-mTORC1 signaling. And we have comfirmed this signaling by western blot. PI3K-AKT-mTORC1 signaling activation results in the increased secretion of PTHrP, and modification of the host osseous environment to promote osteoclast formation and bone colonization. Taken together, these findings provide novel insight into the mechanisms of bone metastasis in $\mathrm{BC}$.

\section{Materials and methods ONCOMINE analysis}

mRNA levels of CENPF in BC were determined through analysis of the ONCOMINE database (http://www. oncomine.org), a publicly accessible online cancer microarray database that facilitates the discovery of genomewide expression analyses. A Students $t$ test was used for the comparison of cancer specimens and normal control datasets. Fold changes were defined as 2 and a $p$-value $\leq 0.01$ was deemed significant.

\section{Microarray analysis}

CENPF gene expression data and the corresponding related clinical parameters were downloaded from the publicly available GEO website (http://www.ncbi. nlm.nih.gov/geo/) including GSE2034 and GSE5034. GSE2034 datasets contained 286 samples, including 180 lymph-node negative relapse free BC patients and 106 lymph-node patients that developed distant metastasis. GDS5306 dataset contained 19 HER2 + human BC brain metastasis patients and 19 HER2 + primary breast tumors.

\section{Prognostic survival analysis}

Clinical prognostic analysis including the overall survival (OS) and relapse free survival (RFS) of CENPF were performed using the Kaplan-Meier method (http://kmplo t.com/analysis/). Kaplan-Meier survival curves, log-rank $P$-values and HR with 95\% confidence intervals were calculated and plotted in $\mathrm{R}$ using Bioconductor packages. Datasets with clinical prognostic information including GSE2034 and GSE39582 were used for prognostic survival analysis. mRNAs in all the datasets were divided into high expression (High) and low expression (Low) groups according to the mean values of CENPF expression.

\section{Gene set enrichment analysis (GSEA)}

Gene set enrichment analysis (GSEA) was used to interpret the gene expression data by determining statistically significant differences in pre-defined gene-sets between biological states. In addition, GSEA can be used to identify the pathways that correlate to gene expression. To probe the biological mechanisms using GSEA software v2.1.0 (Broad Institute, MIT, Cambridge, MA, USA), a 32,619 (gene) $\times 39$ (samples) expression matrix was employed. The predefined gene set 'c2.all.v4.0.symbols. gmt' is one of 7 major collections from the Molecular Signatures Database (MSigDB). A normalized enrichment score (NES) was calculated as the primary GSEA statistic. Gene sets were considered significantly enriched at predefined $\mathrm{p}$-values and FDR $<0.25$.

\section{Cell lines and human tissue samples}

BC 4T1 cells were purchased from the American Type Culture Collection (ATCC) and cells were cultured in RPMI medium 1640 (GIBCO, Gaithersburg, MD, USA) supplemented with $10 \%$ fetal bovine serum (HyClone, Logan, USA) and 1\% penicillin/streptomycin (Invitrogen, Waltham, MA). Cells were grown in 24-well culture dishes (VWR International; Radnor, PA) containing $1.0 \mathrm{ml}$ cell culture medium at $37{ }^{\circ} \mathrm{C}$ in a Hera Cell $5 \%$ $\mathrm{CO}_{2}$ incubator (Thermo Fisher Scientific; Waltham, MA). Culture medium was replaced after 1 day of seeding and then every $48 \mathrm{~h}$ thereafter. A total of 60 formalin-fixed paraffin-embedded BC samples were collected from BC patients who underwent curative-intent surgery without prior radiotherapy and chemotherapy at the Department of Pathology of the Third Affiliated Hospital of Southern Medical University. Informed consent was obtained from each patient on the day of admission. The study was approved by the ethics committee of The Third Affiliated Hospital of Southern Medical University. 


\section{Immunohistochemical analyses}

Primary lesion and bone metastasis samples were fixed in formalin, embedded in paraffin, and analyzed by immunohistochemical analysis. Sections $(2.5 \mu \mathrm{m})$ were deparaffinized and rehydrated, and endogenous peroxidase activity was inhibited with $0.3 \% \mathrm{H}_{2} \mathrm{O}_{2}$-methanol solution. Samples were blocked in 5\% normal goat serum for $1 \mathrm{~h}$, probed with anti-CENPF antibodies (Affinity, DF2310, 1:50 dilution) and anti-PTHrP (ABclonal, A12492, 1:150 dilution) at $4{ }^{\circ} \mathrm{C}$ overnight, and labeled with biotinylated secondary antibodies. The immunoreaction signal was developed with $\mathrm{DAB}$ staining, and slides were counterstained in hematoxylin. Stained tissue sections were viewed under a light microscope (Nikon ECLIPSE Ni-U, Tokyo, Japan).

\section{Animal models}

Six-week-old female BALB/c mice were purchased from the Central Laboratory of Animal Science of Southern Medical University (Guangzhou, China). Mice were provided with standard laboratory diet and drinking water ad libitum, and maintained in a pathogen-free environment at a constant temperature of $23 \pm 1{ }^{\circ} \mathrm{C}$ and humidity of $55 \pm 5 \%$ and with a 12 -h light/12-h dark cycle. All studies for animals were reviewed and approved by the Institutional Animal Care and Use Committee of Southern Medical University. Mice were randomly divided into 2 groups: (1) To investigate the expression of CENPF in primary $\mathrm{BC}$ lesions, $1 \times 10^{5} 4 \mathrm{~T} 1$ cells were inoculated into the mammary fat pad of mice; (2) To investigate CENPF expression in bone metastasis of BC cells, $1 \times 10^{4}$ $4 \mathrm{~T} 1$ cells were inoculated into the left tibia of the mice. After 4 weeks, mice were sacrificed and their organs isolated. Collected organs were fixed in $10 \%$ neutralized formalin solution and paraffin embedded.

\section{siRNA transfection}

CENPF siRNA (5'-GGAGATGCTTCAAACTCAA-3') was obtained from RiboBio (Guangzhou, China) and transfected into 4T1 cells using lipofectamine 3000 (Thermo Fisher Scientific, Rockford, IL, USA). After $48 \mathrm{~h}$, cells were harvested and assessed by western blot analysis.

\section{Western blotting}

Cells were lysed in RIPA buffer (KeyGEN BioTECH) and quantified using Bradford Assays (KeyGEN BioTECH). Lysates were resolved on SDS-PAGE, and transferred to PVDF membranes (Millipore). Membranes were probed with primary antibodies overnight at $4{ }^{\circ} \mathrm{C}$. The primary antibodies included anti-CENPF (Affinity, DF2310, 1:1000 dilution), anti-mTOR (proteintech, 20657-1-AP, 1:1000 dilution), anti-p-mTOR
(Absci, AB11221, 1:1000 dilution), anti-AKT (Cell Signaling, \#4691, 1:1000 dilution), anti-p-AKT (Cell Signaling, \#13038, 1:1000 dilution), anti-PTHrP (ABclonal, A12492, 1:1000 dilution). Membranes were labeled with the appropriate HRP-conjugated secondary antibodies (Fdbio science, FDM007 or FDR007, 1:10,000 dilution) and chemiluminescence was detected using FDbio-Femto ECL western blotting detection reagents (Fdbio science, Hangzhou, China).

\section{Statistical analysis}

Data are presented as the mean \pm standard deviation (SD) from three independent assays using SPSS 22.0 (IBM SPSS Inc. Chicago, IL). A two-tailed Student's t-test (two-tailed) was used to assess differences between the conditions. A $p$-value $<0.05$ was considered statistically significant.

\section{Results}

\section{CENPF is overexpressed in breast and lung cancer}

From ONCOMINE analysis, CENPF mRNA expression was significantly higher in $\mathrm{BC}$ samples across the 14 datasets in different cancer types (Table 1 and Fig. 1a, b). CENPF transcripts were elevated by $\geq 5.3$-fold in $\mathrm{BC}$ samples compared to normal tissue. The samples included 593 samples derived from the TCGA (the Cancer Genome Atlas) database (Fig. 2a-d). In previous studies [20], CENPF was $\geq 3.1$ fold elevated in BC samples compared to normal tissue (Fig. 2e, f). Similarly, CENPF was $\geq 2.0$ fold elevated in 2136 BC samples [21] (Fig. 2g-1). In other studies [22, 23], CENPF was 5.2 fold (total samples $=47$ ) and 7.1 fold higher in $\mathrm{BC}$ samples (Fig. 2m, n) compared to normal tissue. To further determine the role of CENPF in BC, 60 $\mathrm{BC}$ samples and paired normal tissue were collected and assessed by immunohistochemistry (IHC) staining. This confirmed that CENPF is expressed to higher levels in $\mathrm{BC}(42 / 60,70 \%)$ compared to normal tissues $(20 / 60,33.3 \%)(p<0.01)$ (Fig. 3a, b).

Lung cancer is also prone to bone metastasis. Our analysis also demonstrated significantly higher CENPF expression in lung cancer versus normal samples (Additional file 1: Fig. S1A-I). In the datasets reported by Bhattacharjee and coworkers [24] from 186 samples, CENPF was 24.5 fold higher in lung cancer samples compared to normal tissue (Additional file 1: Fig. S1A).

\section{High CENPF mRNA expression correlates with poor OS and RFS in BC patients}

Kaplan-Meier analysis demonstrated that high CENPF mRNA expression is significantly associated with shorter 
Table 1 The situation of each sub-database

\begin{tabular}{|c|c|c|c|c|c|}
\hline Database & $P$ value & Fold change & Sample size & $\begin{array}{l}\text { Sample size } \\
\text { of normal }\end{array}$ & $\begin{array}{l}\text { Sample size } \\
\text { of breast } \\
\text { cancer }\end{array}$ \\
\hline \multirow[t]{4}{*}{ TCGA breast } & $1.13 \mathrm{E}-21$ & 6.980 & 97 & 61 & 36 \\
\hline & $2.23 \mathrm{E}-35$ & 5.980 & 137 & 61 & 76 \\
\hline & $1.91 \mathrm{E}-44$ & 6.503 & 450 & 61 & 389 \\
\hline & $1.37 \mathrm{E}-5$ & 5.380 & 68 & 61 & 7 \\
\hline \multirow[t]{2}{*}{ Ma breast 4} & $3.50 E-6$ & 3.188 & 23 & 14 & 9 \\
\hline & $1.74 \mathrm{E}-5$ & 3.568 & 23 & 14 & 9 \\
\hline \multirow[t]{6}{*}{ Curtis breast } & $4.86 \mathrm{E}-106$ & 2.866 & 1700 & 144 & 1556 \\
\hline & $1.49 \mathrm{E}-15$ & 4.511 & 176 & 144 & 32 \\
\hline & $8.50 E-39$ & 2.417 & 292 & 144 & 148 \\
\hline & $5.23 \mathrm{E}-7$ & 2.072 & 165 & 144 & 21 \\
\hline & $1.15 E-25$ & 2.357 & 234 & 144 & 90 \\
\hline & $6.91 \mathrm{E}-5$ & 2.056 & 158 & 144 & 14 \\
\hline Richardson breast 2 & $2.45 E-9$ & 7.131 & 47 & 7 & 40 \\
\hline Zhao breast & $5.49 E-5$ & 5.244 & 41 & 3 & 38 \\
\hline
\end{tabular}

a

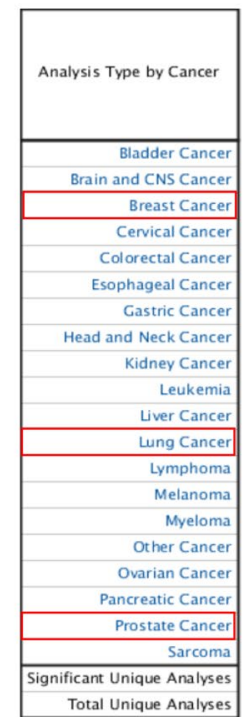

Disease Summary for CENPF
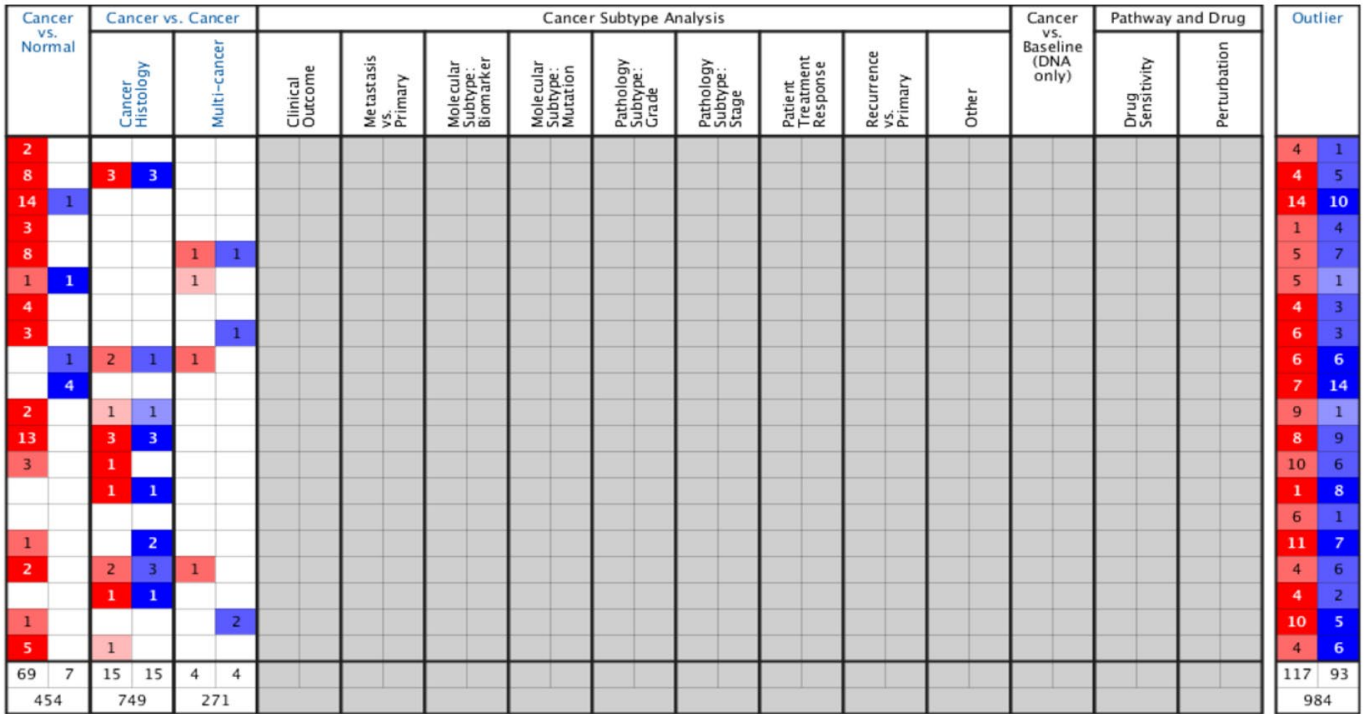

\section{$\begin{array}{lll}10 & 5 & 1\end{array}$

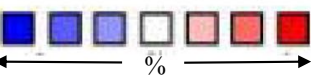

b

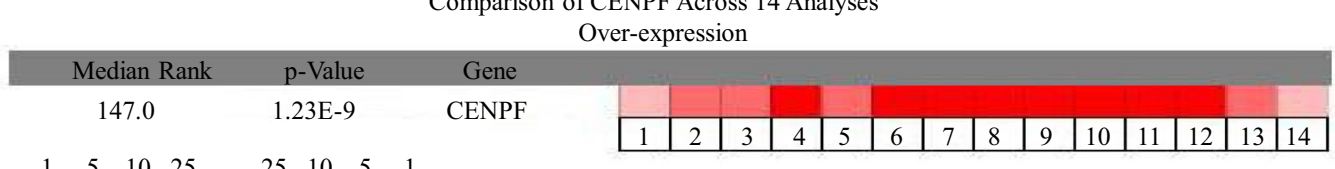

$\begin{array}{llllllll}1 & 5 & 10 & 25 & 25 & 10 & 5 & 1\end{array}$ $\square \square \square \square \square \square \square \square \square \square$ Not measured

Fig. 1 mRNA expression of CENPF in different tumors. Graphs show the number of datasets with statistically significant mRNA overexpression (red) or down-regulation (blue) of the target gene (cancer vs. normal tissue and cancer vs. cancer). $p$ value thresholds were 0.01. Numbers in each cell indicate the number of analyses that met the threshold within each analysis and cancer type. Cell colors demonstrate the best gene rank percentile for analyses. CENPF was compared across 14 analyses. Values of the genes indicate the median rank. $p$ values were assessed for each gene and for each median-ranked analysis $(\mathbf{a}, \mathbf{b})$ 


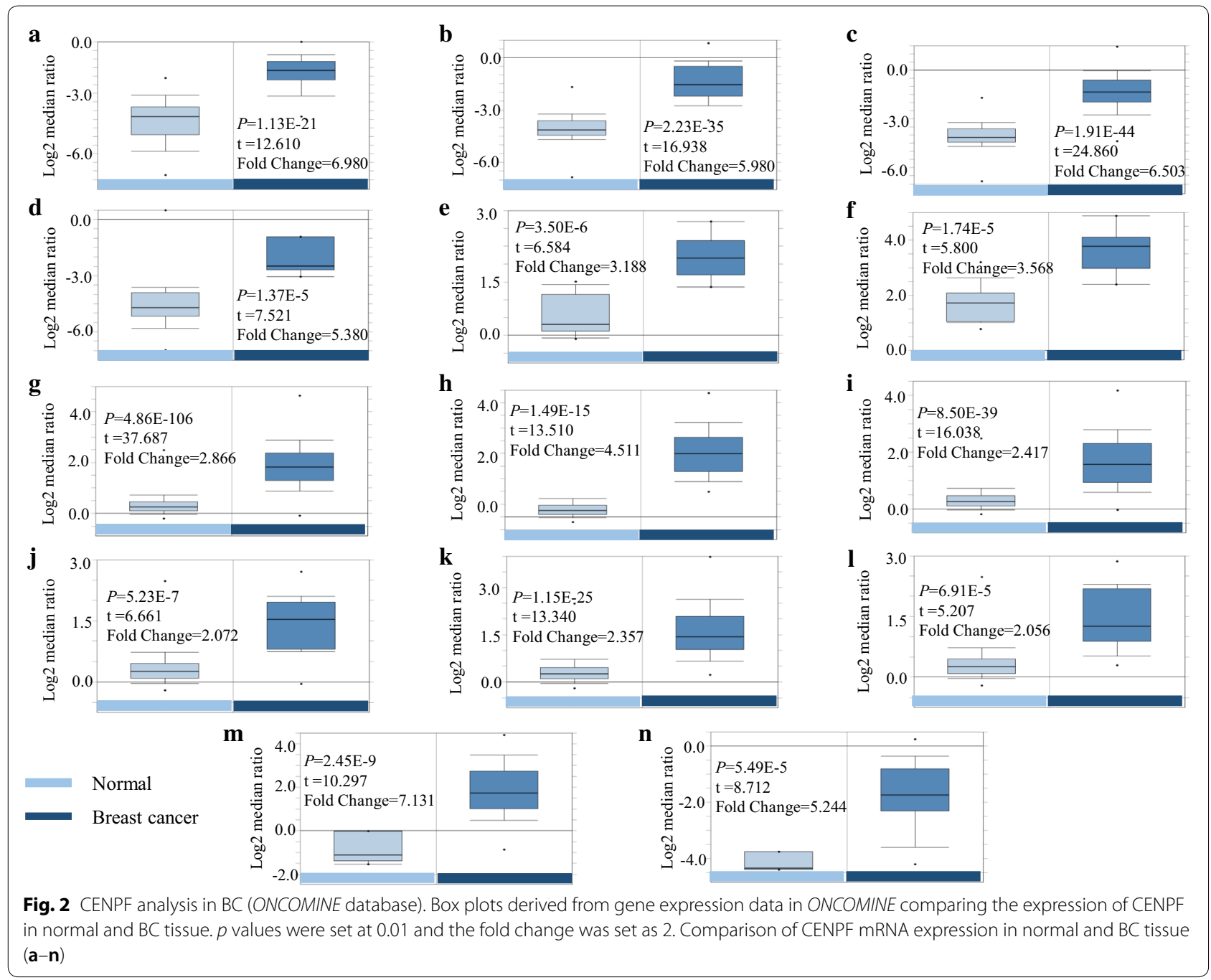

OS and RFS in $\mathrm{BC}(\mathrm{HR}=1.61(1.3-2), p=1.3 \mathrm{e}-05)$ and $(\mathrm{HR}=1.39$ (1.25-1.55), $p=3 \mathrm{e}-09$ respectively), (Fig. 3c, d). The analysis of GSE2034 (from GEO datasets) demonstrated that high CENPF mRNA expression led to a poor prognosis $(p=0.0038)$ (Fig. 3e). Similarly, high CENPF mRNA expression was associated with decreased survival in lung cancer $(\mathrm{HR}=1.57(1.38-1.78), p=3.4 \mathrm{e}-12)$ (Fig. 3f). Of note, high CENPF expression significantly correlated to shorter OS and RFS in BC patients. This indicated a role for CENPF in the prognosis of $\mathrm{BC}$.

\section{CENPF expression is higher in bone metastasis in BC than that in primary $B C$ lesions and other organs}

The GSE2034 is a published dataset consisting of 180 $\mathrm{BC}$ specimens without bone metastasis, $69 \mathrm{BC}$ specimens with bone metastasis, and $37 \mathrm{BC}$ specimens with other organ metastasis (including lung and brain metastasis). We compared the mRNA expression of CENPF in the 3 types of specimen and found that the expression of CENPF in bone metastasis is higher than primary BC lesions, but does not differ between primary $\mathrm{BC}$ lesions and the metastasis of other organs (Fig. 4a). Through GDS5306 analysis, published datasets consisting of 19 brain metastasis specimens and matched primary breast tumor specimens of $19 \mathrm{BC}$ patients, we also found that the mRNA expression of CENPF does not differ between primary breast tumors and brain metastasis tissue (Fig. 4b).

In the four $4 \mathrm{~T} 1$ primary $\mathrm{BC}$ models and four bone metastasis models of BALB/C mice, CENPF was expressed to higher levels in $75 \%$ (3/4) of bone metastasis tissue samples compared to primary lesions and lung metastasis tissues. Interestingly, the expression of PTHrP in these three different tissues showed the same trend as CENPF. However, no significant differences were observed between primary lesions and lung metastatic tissue (Fig. 4d). These results further demonstrate 

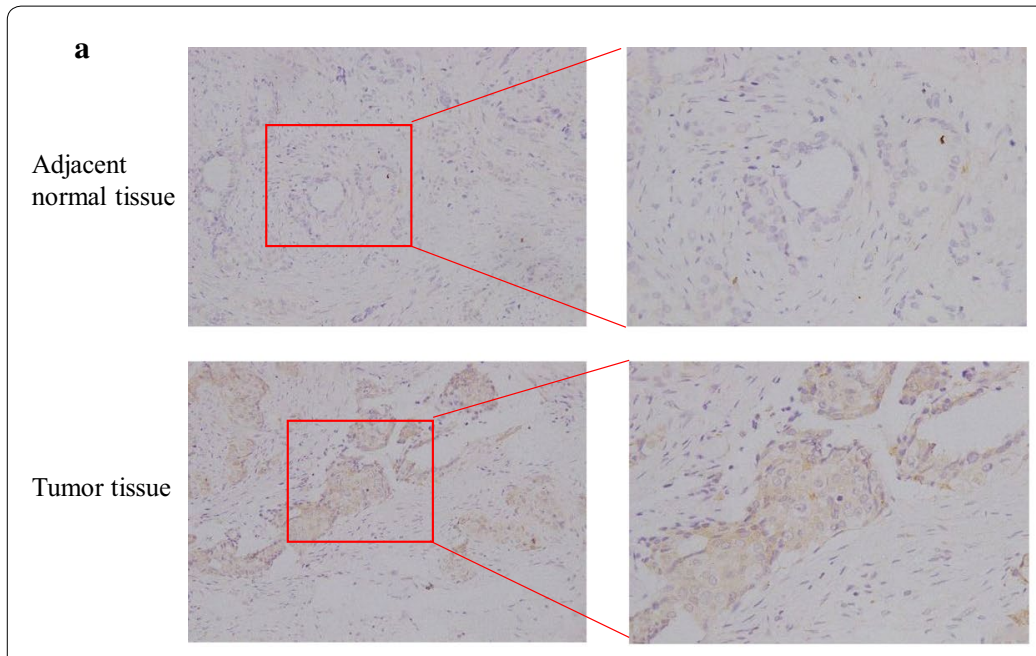

b

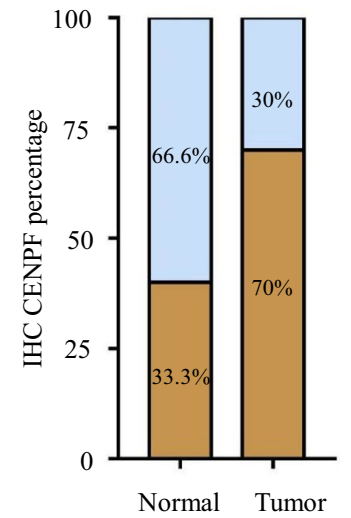

c

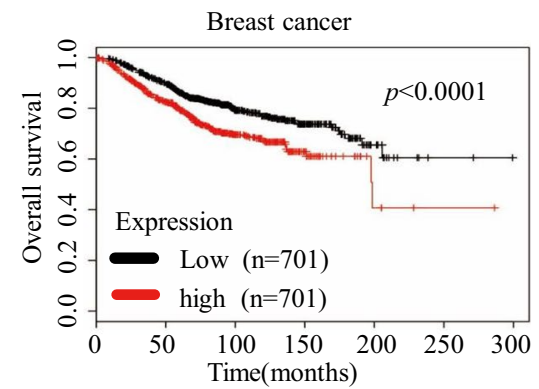

d

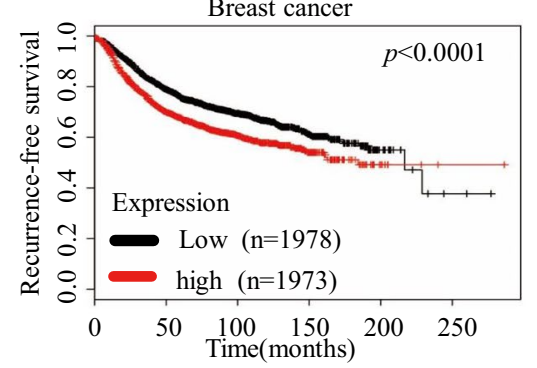

f
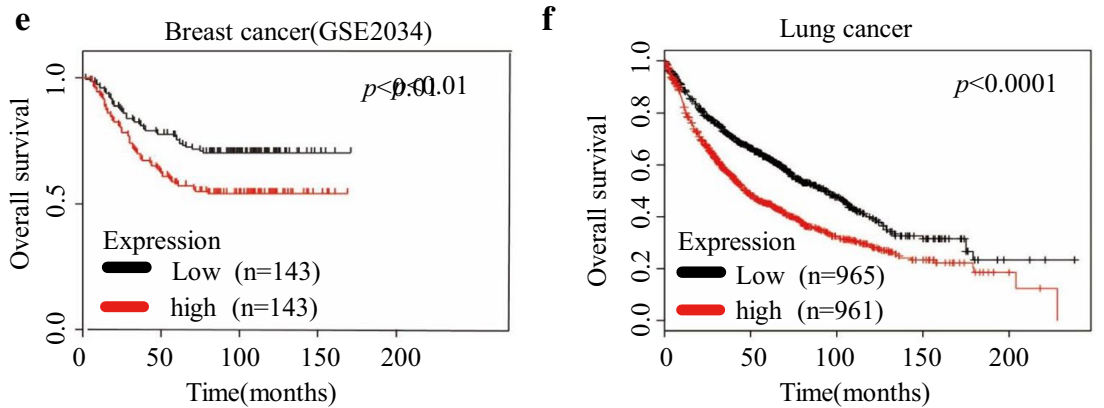

Fig. 3 CENPF in tumor tissue and adjacent histologically normal tissue of BC patients $(\times 400)$ (a). Percentage of CENPF IHC in BC and matched adjacent normal tissue. $\mathbf{b}$ High CENPF mRNA levels were associated with shorter OS (c), reduced RFS (d), and shorter OS in BC patients with high CENPF mRNA expression (e). High mRNA levels of CENPF were associated with shorter OS in lung cancer patients ( $\mathbf{f}$ )

that CENPF plays an important role in bone metastasis during BC. In addition, there may be a close correlation between CENPF and PTHrP in breast cancer bone metastasis.

\section{GSEA reveals a potential role of CENPF in oncogenic Signaling during tumor metastasis}

To identify the cellular mechanisms by which CENPF influences tumor development, gene set enrichment analysis (GSEA) was used to compare the gene expression profiles of $\mathrm{CENPF}^{\text {low }}$ and $\mathrm{CENPF}^{\text {high }}$ in $\mathrm{BC}$ specimens. The GSE2034 database contains 286 BC tissues divided into CENPF $^{\text {low }}(n=143)$ and CENPF ${ }^{\text {high }}$ $(n=143)$ groups based on the median expression level of CENPF. GSEA analysis revealed a significant association between CENPF and cell cycle regulation, P53, and PI3K-AKT-mTOR signaling suggesting a role for these pathways in the metastatic activity of CENPF (Fig. 5ad). As expected, the activation of AKT/mTOR signaling pathway and the expression of PTHrP were dramatically inhibited in 4T1 cells with silenced CENPF (Fig. 5e).

\section{Discussion}

Previous studies have demonstrated that the overexpression of CENPF plays an important role in prostate cancer development $[25,26]$. CENPF has been shown to be a synergistic master regulator of prostate cancer malignancy and a prognostic indicator of poor survival and metastasis [18]. However, the roles of CENPF in the other cancers are less well understood and the functions of CENPF remain undefined. In this study, we show that CENPF is upregulated in BC tissue, particularly in BC bone metastatic lesions, which positively correlates with poor survival in human BC patients. Furthermore, we performed GSEA to explore the potential mechanisms of CENPF bone metastasis. 


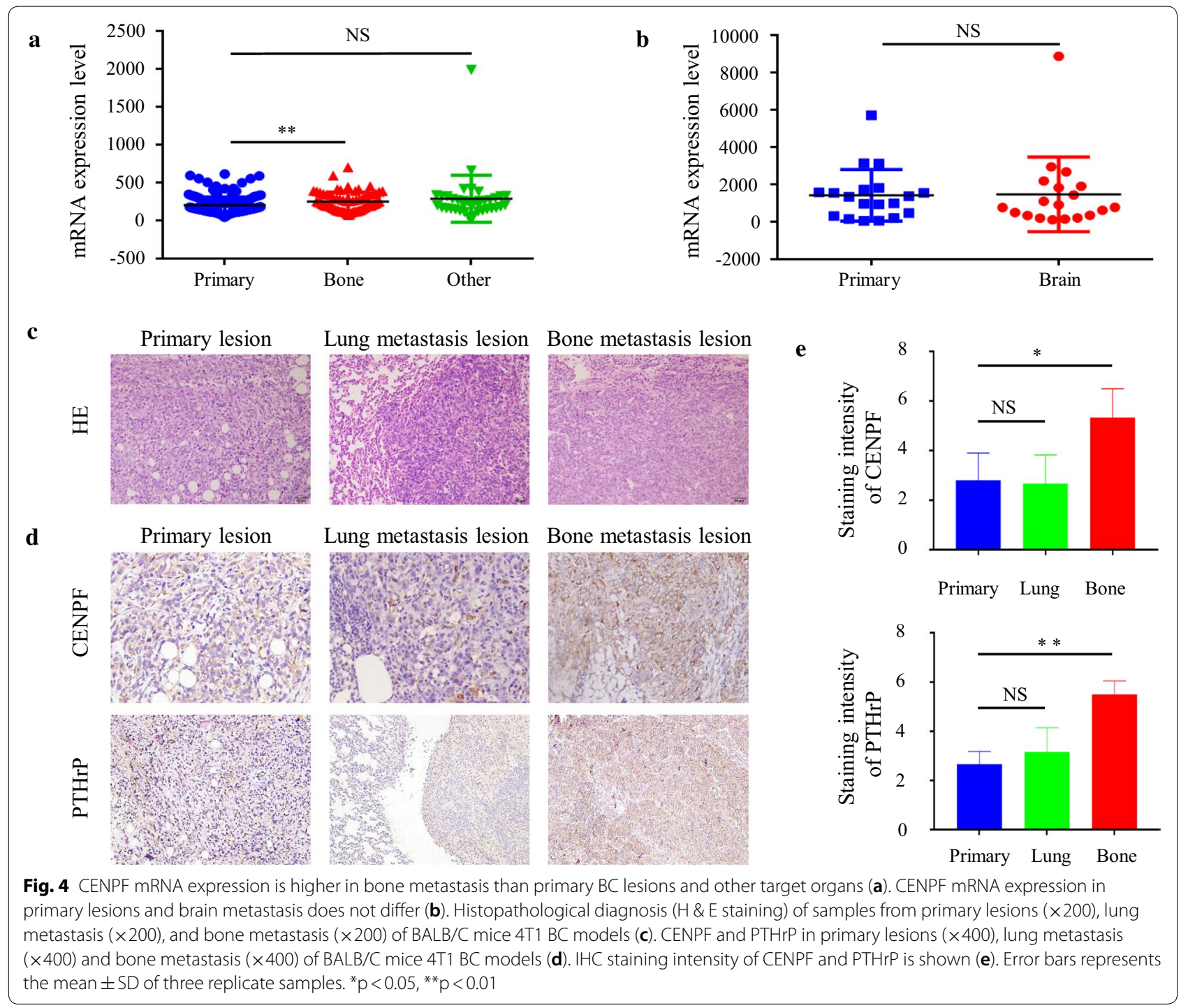

Using bioinformatics and experimental analysis, CENPF was found to play a vital role in BC progression and bone metastasis. CENPF was found to be frequently upregulated in $\mathrm{BC}$ and other cancers prone to bone metastasis including lung cancer and prostate cancer (Figs. 1, 2). To determine the expression of CENPF in human BC tissue, we performed IHC staining of CENPF in $60 \mathrm{BC}$ tissues. The staining intensity of CENPF was predominantly in $\mathrm{BC}$ tissues, and weak staining was detected in normal adjacent tissue (Fig. 3a, b). Secondly, we performed Kaplan-Meier analyses to show that CENPF is a prognostic marker for clinical outcomes (Fig. 3c-f). Thus, CENPF may function as a tumor promoter during $\mathrm{BC}$ progression.

Additionally, we used GEO datasets containing primary $\mathrm{BC}$ lesions and distant metastatic lesions to perform microarray analysis. We found that CENPF expression is higher in bone metastatic lesions compared to primary $\mathrm{BC}$ lesions and other distant organs (Fig. 4a, b). As CENPF is highly expressed in BC, particularly in bone metastatic lesions, these results are consistent with its reported role in bone metastasis in prostate and lung cancer cases.

Given these findings, we hypothesized that CENPF is closely related to bone metastasis in BC. Furthermore, we performed IHC staining of CENPF in BC primary lesions, lung metastasis lesions, and bone metastasis lesions, which were collected from our animal model. We found that the intensity of CENPF staining was higher in primary lesions and lung metastasis lesions (Fig. 4d, e). These results confirm that CENPF promotes $\mathrm{BC}$ bone metastasis.

Since bone metastasis is the leading cause of $\mathrm{BC}$ related death [27, 28], understanding the molecular role of 


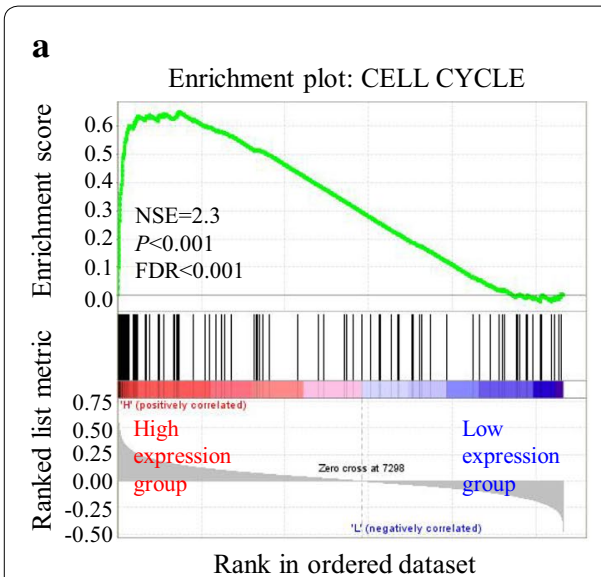

c

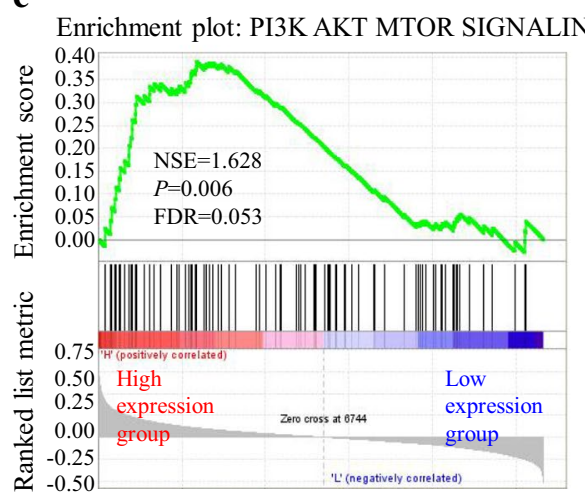

b

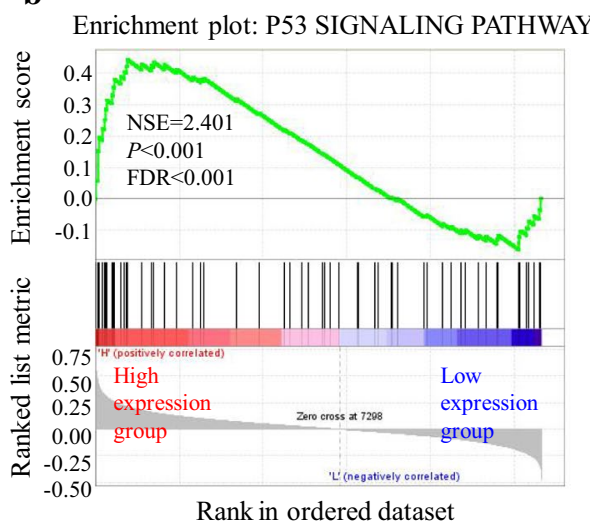

d

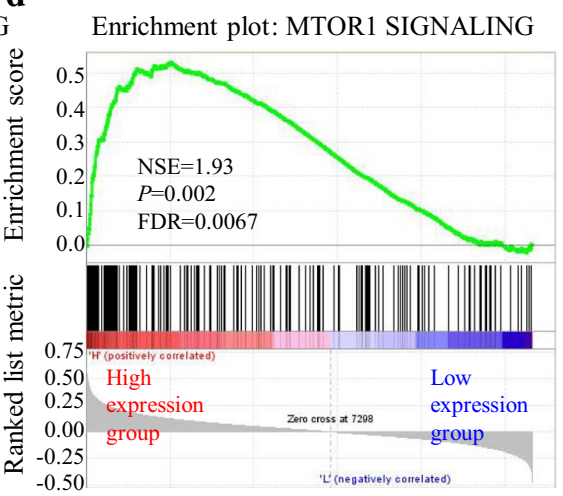

e control

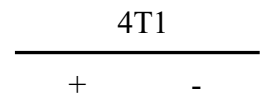

si-CENPF

p-mTOR

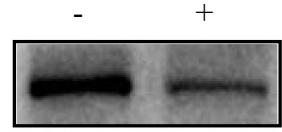

1.12 0.69

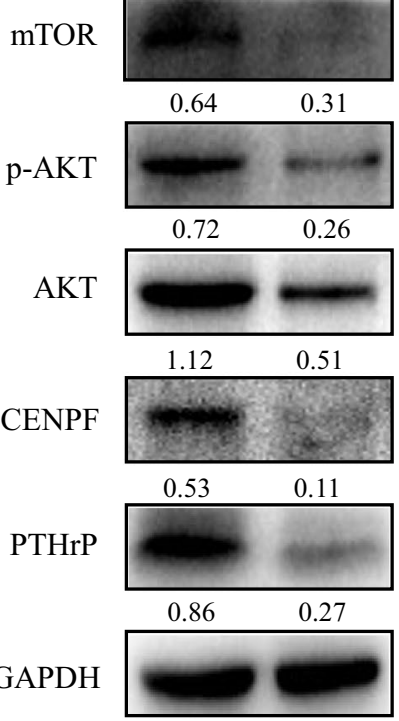

Fig. 5 GSEA reveals potential downstream signaling of CENPF. GSEA results showed that the cell cycle (a), P53 signaling pathways (b), PI3K/AKT/ mTOR signaling $(\mathbf{c})$, and mTORC1 pathways $(\mathbf{d})$ were enriched in the high CENPF expression group. Top panels indicate the enrichment scores for each gene. Bottom panels show the ranking metrics of each gene. Y-axis: ranking metric values; X-axis: ranks for all genes. NES: normalized enrichment score. CENPF silencing reduced the expression of AKT, p-AKT, mTOR, p-MTOR and PTHrP as assessed by western blot analysis (e)

CENPF driven bone metastasis can direct future therapeutic strategies. The development of $\mathrm{BC}$ bone metastasis is a complex process involving crosstalk between disseminated BC cells and bone-derived molecules, leading to deregulated signaling pathways that are critical for normal bone remodeling processes [4]. Herein, we performed GSEA to explore the potential mechanisms of CENPF driven $\mathrm{BC}$ progression and bone metastasis. Our results showed that the CENPF expression was significantly associated with P53, cell-cycle progression and the G2 M-Checkpoint. Previous studies have shown that CENPF is a component of the nuclear matrix during the G2 stage of interphase, where in gradually accumulates during the cell cycle, reaching peak levels in the G2/M phase, and is degraded upon the completion of mitosis [29]. Notably, CENPF expression was also enriched in the PI3K-AKT-mTOR and mTORC1 signaling pathways and we confirmed that the activation of AKT/mTOR signaling pathway and the expression of PTHrP were dramatically inhibited in 4T1 cells with silenced CENPF (Fig. 5).
As we have discovered, knocking down CENPF not only inhibits the synthesis of mTOR and AKT, but also inhibits their phosphorylation (Fig. 5e).

PI3K/AKT/mTOR signaling is an important intracellular pathway that is frequently activated in diverse cancers. PI3K/AKT/mTOR regulates cell proliferation, differentiation, cellular metabolism and cancer cell survival. PI3K/AKT/mTOR activation promotes tumor development as drug resistance [30, 31]. Cancer bone metastasis is a complex, multistage process that includes local invasion, intravasation, survival in the circulation, extravasation, and colonization [32, 33]. Within this process are various molecules including parathyroid hormone-related peptide (PTHrP) $[11,12]$. Previous studies have demonstrated that the downstream S6 kinase 1 of mTORC1 interacts with and phosphorylates Gli2, permitting its release and the subsequent transcriptional activation of PTHrP, a key regulator of bone development [34]. PTHrP participates in bone remodeling through osteoclastogenesis 


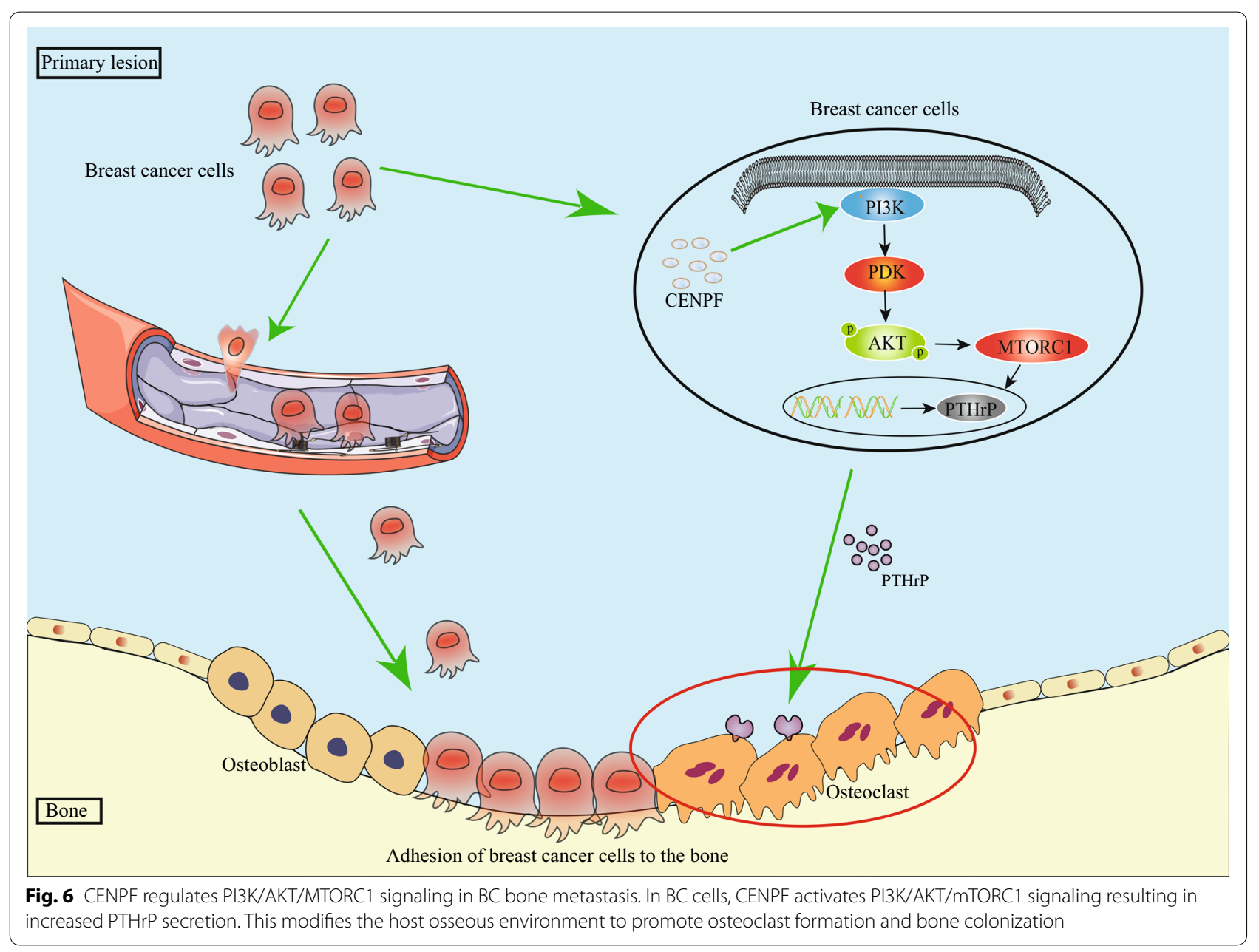

and facilitates tumor localization and growth in the bone [35]. CENPF overexpression in BC may thus activate $\mathrm{mTORC} 1$ to regulate $\mathrm{PTHrP}$, which modifies the host osseous environment to promote osteoclast formation and bone colonization [36].

In summary, we have revealed the metastatic promoter function of CENPF in $\mathrm{BC}$ progression and bone metastasis. High CENPF expression in $\mathrm{BC}$ activates mTORC1 and regulates PTHrP, which modifies the bone microenvironment permitting an ease of transfer of $\mathrm{BC}$ cells to the bone (Fig. 6). However, some limitations remain as the potential molecular mechanisms were not verified experimentally. We envision that therapeutic intervention centered on inhibiting CENPF function could be useful for the prevention of $\mathrm{BC}$ bone metastasis.

\section{Conclusion}

Our results demonstrated that CENPF promotes BC bone metastasis by activating the PI3K-AKT-mTORC1 signaling pathway. CENPF may serve as a novel therapeutic, diagnostic, and/or prognostic target in breast cancer treatment.

\section{Supplementary information}

Supplementary information accompanies this paper at https://doi. org/10.1186/s12935-019-0986-8.

Additional file 1: Figure S1. CENPF in lung cancer (ONCOMINE database). Box plots derived from gene expression data in ONCOMINE comparing the expression of the CENPF in normal and LC tissue. $p$-values were set at 0.01 and the fold change was defined as 2. Comparison of CENPF mRNA expression in normal and lung cancer tissue (A-L).

\section{Abbreviations}

CENPF: centromere protein F; BC: breast cancer; IHC: immunohistochemical; HE: hematoxylin; GEO: the Gene Expression Omnibus; OS: overall survival; RFS: relapse free survival; GSEA: gene set enrichment analysis; PTHrP: parathyroid hormone-related peptide; p: phosphor; PI3K: phosphatidylinositol 3-kinase; AKT: serine-threonine protein kinase; mTOR: mechanistic target of rapamycin kinase; mTORC1: mechanistic target of rapamycin kinase complex 1; BM: bone metastasis; IL-8: interleukin 8; FOXM1: forkhead box protein M1; VCAM-1:

vascular cell adhesion molecule 1; HCC: hepatocellular carcinoma; COUP-TFIl: chicken ovalbumin upstream promoter transcription factor 2; PC: prostatic cancer; NES: normalized enrichment score; FDR: false discovery rate; MSigDB: 
Molecular Signatures Database; ATCC: the American Type Culture Collection; SD: standard deviation; HR: hazard ratio.

\section{Acknowledgements}

The authors thank Professor Li Liang of the Key Laboratory of Molecular Tumor Pathology in Guangdong Province for her instructions on pathological analyses.

\section{Authors' contributions}

Study design: LX, DY, SJ, HJ and LJ. Study conduct: SJ, HJ and L. Data collection: SJ, ZK, GY and SZ. Data analysis: SZ, SJ, GY and DY. Data interpretation: DY, DY, SJ, HJ, LJ, ZK and SZ. Drafting manuscript: SJ, HJ, LJ, LL, DY and LX. Revising manuscript: SJ, LL, DY and LX. All authors read and approved the final manuscript.

\section{Funding}

Not applicable.

\section{Availability of data and materials}

Datasets used and/or analyzed data are available from the corresponding author upon reasonable request.

\section{Ethics approval and consent to participate}

This study was approved by the ethics committee of The Third Affiliated Hospital of Southern Medical University.

\section{Consent for publication}

Not applicable.

\section{Competing interests}

The authors declare that they no competing interests.

\begin{abstract}
Author details
${ }^{1}$ Department of General Surgery, The Third Affiliated Hospital of Southern Medical University, 183 West Zhongshan Avenue, Guangzhou 510630, Guangdong, China. ${ }^{2}$ Department of Metabolic Surgery, General Hospital of Guangzhou Military Command, Southern Medical University, Guangzhou 510515, China. ${ }^{3}$ Nursing Department, The Third Affiliated Hospital of Southern Medical University, 183 West Zhongshan Avenue, Guangzhou 510630, Guangdong, China.
\end{abstract}

Received: 30 March 2019 Accepted: 30 September 2019 Published online: 11 October 2019

\section{References}

1. Ban KA, Godellas CV. Epidemiology of breast cancer. Surg Oncol Clin N Am. 2014;23(3):409-22.

2. Xiong Z, Deng G, Huang X, Li X, Xie X, Wang J, Shuang Z, Wang X. Bone metastasis pattern in initial metastatic breast cancer: a population-based study. Cancer Manag Res. 2018;10:287-95.

3. von Moos R, Body JJ, Egerdie B, Stopeck A, Brown J, Fallowfield L, Patrick DL, Cleeland C, Damyanov D, Palazzo FS, et al. Pain and analgesic use associated with skeletal-related events in patients with advanced cancer and bone metastases. Support Care Cancer. 2016;24(3):1327-37.

4. Brook N, Brook E, Dharmarajan A, Dass CR, Chan A. Breast cancer bone metastases: pathogenesis and therapeutic targets. Int J Biochem Cell Biol. 2018;96:63-78.

5. Kozlow W, Guise TA. Breast cancer metastasis to bone: mechanisms of osteolysis and implications for therapy. J Mammary Gland Biol Neoplasia. 2005;10(2):169-80.

6. Lu X, Kang Y. Organotropism of breast cancer metastasis. J Mammary Gland Biol Neoplasia. 2007;12(2-3):153-62.

7. Hofbauer LC, Rachner T, Singh SK. Fatal attraction: why breast cancer cells home to bone. Breast Cancer Res. 2008;10(1):101.

8. Guise TA, Mohammad KS, Clines G, Stebbins EG, Wong DH, Higgins LS, Vessella R, Corey E, Padalecki S, Suva L, et al. Basic mechanism responsible for osteolytic and osteoblastic bone metastases. Clin Cancer Res. 2006;12(20 Pt 2):6213s-6s.

9. Krzeszinski JY, Wan Y. New therapeutic targets for cancer bone metastasis. Trends Pharmacol Sci. 2015;36(6):360-73.

10. Yao B, Wang J, Qu S, Liu Y, Jin Y, Lu J, Bao Q, Li L, Yuan H, Ma C. Upregulated osterix promotes invasion and bone metastasis and predicts for a poor prognosis in breast cancer. Cell death \& disease. 2019:10(1):28.

11. Guise TA, Yin JJ, Taylor SD, Kumagai Y, Dallas M, Boyce BF, Yoneda T, Mundy GR. Evidence for a causal role of parathyroid hormone-related protein in the pathogenesis of human breast cancer-mediated osteolysis. J Clin Invest. 1996;98(7):1544-9.

12. Bendre MS, Gaddy-Kurten D, Mon-Foote T, Akel NS, Skinner RA, Nicholas RW, Suva LJ. Expression of interleukin 8 and not parathyroid hormonerelated protein by human breast cancer cells correlates with bone metastasis in vivo. Cancer Res. 2002;62(19):5571-9.

13. Lu X, Mu E, Wei Y, Riethdorf S, Yang Q, Yuan M, Yan J, Hua Y, Tiede BJ, Lu $X$, et al. VCAM-1 promotes osteolytic expansion of indolent bone micrometastasis of breast cancer by engaging alpha4beta1-positive osteoclast progenitors. Cancer Cell. 2011;20(6):701-14.

14. Landberg G, Erlanson M, Roos G, Tan EM, Casiano CA. Nuclear autoantigen p330d/CENP-F: a marker for cell proliferation in human malignancies. Cytometry. 1996;25(1):90-8.

15. Kim HE, Kim DG, Lee KJ, Son JG, Song MY, Park YM, Kim JJ, Cho SW, Chi $\mathrm{SG}$, Cheong HS, et al. Frequent amplification of CENPF, GMNN and CDK13 genes in hepatocellular carcinomas. PLoS ONE. 2012;7(8):e43223.

16. Varis A, Salmela AL, Kallio MJ. Cenp-F (mitosin) is more than a mitotic marker. Chromosoma. 2006;115(4):288-95.

17. Sun B, Lin G, Ji D, Li S, Chi G, Jin X. Dysfunction of sister chromatids separation promotes progression of hepatocellular carcinoma according to analysis of gene expression profiling. Front Physiol. 2018;9:1019.

18. Aytes A, Mitrofanova A, Lefebvre C, Alvarez MJ, Castillo-Martin M, Zheng T, Eastham JA, Gopalan A, Pienta KJ, Shen MM, et al. Cross-species regulatory network analysis identifies a synergistic interaction between FOXM1 and CENPF that drives prostate cancer malignancy. Cancer Cell. 2014;25(5):638-51.

19. Lin SC, Kao CY, Lee HJ, Creighton CJ, Ittmann MM, Tsai SJ, Tsai SY, Tsai MJ. Dysregulation of miRNAs-COUP-TFII-FOXM1-CENPF axis contributes to the metastasis of prostate cancer. Nat Commun. 2016;7:11418.

20. Ma XJ, Dahiya S, Richardson E, Erlander M, Sgroi DC. Gene expression profiling of the tumor microenvironment during breast cancer progression. Breast Cancer Res. 2009;11(1):R7.

21. Curtis C, Shah SP, Chin SF, Turashvili G, Rueda OM, Dunning MJ, Speed D, Lynch AG, Samarajiwa S, Yuan Y, et al. The genomic and transcriptomic architecture of 2,000 breast tumours reveals novel subgroups. Nature. 2012;486(7403):346-52.

22. Richardson AL, Wang ZC, De Nicolo A, Lu X, Brown M, Miron A, Liao X, Iglehart JD, Livingston DM, Ganesan S. X chromosomal abnormalities in basal-like human breast cancer. Cancer Cell. 2006;9(2):121-32.

23. Zhao H, Langerod A, Ji Y, Nowels KW, Nesland JM, Tibshirani R, Bukholm IK, Karesen R, Botstein D, Borresen-Dale AL, et al. Different gene expression patterns in invasive lobular and ductal carcinomas of the breast. Mol Biol Cell. 2004;15(6):2523-36.

24. Bhattacharjee A, Richards WG, Staunton J, Li C, Monti S, Vasa P, Ladd C, Beheshti J, Bueno R, Gillette M, et al. Classification of human lung carcinomas by mRNA expression profiling reveals distinct adenocarcinoma subclasses. Proc Natl Acad Sci USA. 2001;98(24):13790-5.

25. Testa JR, Zhou JY, Bell DW, Yen TJ. Chromosomal localization of the genes encoding the kinetochore proteins CENPE and CENPF to human chromosomes 4q24-> q25 and 1q32-> q41, respectively, by fluorescence in situ hybridization. Genomics. 1994;23(3):691-3.

26. Laoukili J, Kooistra MR, Bras A, Kauw J, Kerkhoven RM, Morrison A, Clevers $\mathrm{H}$, Medema $\mathrm{RH}$. FoxM1 is required for execution of the mitotic programme and chromosome stability. Nat Cell Biol. 2005;7(2):126-36.

27. Jemal A, Siegel R, Ward E, Hao Y, Xul J, Thun MJ. Cancer statistics, 2009. CA Cancer J Clin. 2009;59(4):225-49.

28. Chen W, Zheng $R$, Baade PD, Zhang S, Zeng H, Bray F, Jemal A, Yu XQ, He J. Cancer statistics in China, 2015. CA Cancer J Clin. 2016;66(2):115-32.

29. Liao H, Winkfein RJ, Mack G, Rattner JB, Yen TJ. CENP-F is a protein of the nuclear matrix that assembles onto kinetochores at late $\mathrm{G} 2$ and is rapidly degraded after mitosis. J Cell Biol. 1995;130(3):507-18. 
30. Fruman DA, Chiu H, Hopkins BD, Bagrodia S, Cantley LC, Abraham RT. The PI3K pathway in human disease. Cell. 2017;170(4):605-35.

31. Sathe A, Nawroth R. Targeting the PI3K/AKT/mTOR pathway in Bladder Cancer. Methods Mol Biol. 2018;1655:335-50.

32. Burris HA 3rd. Overcoming acquired resistance to anticancer therapy: focus on the PI3K/AKT/mTOR pathway. Cancer Chemother Pharmacol. 2013;71(4):829-42.

33. Jin K, Li T, van Dam H, Zhou F, Zhang L. Molecular insights into tumour metastasis: tracing the dominant events. J Pathol. 2017:241(5):567-77.

34. Yan B, Zhang Z, Jin D, Cai C, Jia C, Liu W, Wang T, Li S, Zhang H, Huang $B$, et al. mTORC1 regulates PTHrP to coordinate chondrocyte growth, proliferation and differentiation. Nat Commun. 2016;7:11151.
35. Sohail A, Sherin L, Butt SI, Javed S, Li Z, lqbal S, Be'g OA. Role of key players in paradigm shifts of prostate cancer bone metastasis. Cancer Manag Res. 2018;10:1619-26.

36. Yin JJ, Selander K, Chirgwin JM, Dallas M, Grubbs BG, Wieser R, Massague J, Mundy GR, Guise TA. TGF-beta signaling blockade inhibits PTHrP secretion by breast cancer cells and bone metastases development. J Clin Invest. 1999;103(2):197-206.

\section{Publisher's Note}

Springer Nature remains neutral with regard to jurisdictional claims in published maps and institutional affiliations.
Ready to submit your research? Choose BMC and benefit from:

- fast, convenient online submission

- thorough peer review by experienced researchers in your field

- rapid publication on acceptance

- support for research data, including large and complex data types

- gold Open Access which fosters wider collaboration and increased citations

- maximum visibility for your research: over $100 \mathrm{M}$ website views per year

At BMC, research is always in progress.

Learn more biomedcentral.com/submissions 